\title{
Time resolved radiated power during tokamak disruptions and spectral averaging of AXUV photodiode response in DIII-D
}

\author{
D. S. Gray, ${ }^{\text {a) }}$ S. C. Luckhardt, L. Chousal, and G. Gunner \\ Center for Energy Research, Fusion Division, University of California at San Diego MC 0417, \\ 9500 Gilman Drive, La Jolla, California 92093
}

A. G. Kellman

General Atomics, 3550 General Atomics Court, San Diego, California 92121

D. G. Whyte

University of Wisconsin-Madison, 1500 Engineering Drive, Madison, Wisconsin 53706

(Received 18 October 2002; accepted 24 November 2003)

\begin{abstract}
Silicon absolute extreme ultraviolet (AXUV) photodiodes have been employed in a disruption radiometer diagnostic for measurement of radiant power in the DIII-D tokamak with a $170 \mathrm{kHz}$ bandwidth. This is motivated by a need to improve the understanding of radiative processes in tokamak disruptions. The diagnostic described in this article has a single line of sight though the central plasma. Accounting for the photon energy dependence of the AXUV photodiode responsivity is made possible by optical filtering, with the aid of spectra from an extreme ultraviolet survey spectrometer. The appropriate effective responsivity for interpretation of the data is lower than the nominal value typically used for the detector. In the current quench phase of disruptions, it is less than half the nominal value. Comparisons with results from a foil bolometer find good agreement. (C) 2004 American Institute of Physics. [DOI: 10.1063/1.1642745]
\end{abstract}

\section{INTRODUCTION}

As a candidate concept for a fusion reactor, the tokamak must overcome the problem of the sudden plasma terminations known as disruptions. ${ }^{1}$ Disruptions in medium-size tokamaks, such as DIII-D, are characterized by a loss of the stored thermal energy on time scales that can be as short as $10^{-4} \mathrm{~s}$ (thermal quench or TQ), followed by a dissipation on a $10^{-3} \mathrm{~s}$ time scale of the energy stored in the poloidal magnetic field (current quench or CQ). Progress in the understanding of these events has been hampered by a shortage of appropriate diagnostics. While a number of diagnostics are fast enough to time resolve disruptions, and some diagnostics produce measurements that are valid and potentially useful during disruptions, few diagnostics meet both of these conditions.

Radiant power is one quantity of central importance in understanding the TQ phase of disruptions. Silicon "absolute extreme ultraviolet" (AXUV) photodiodes ${ }^{2}$ have been applied to fusion plasma experiments in recent years, ${ }^{3-6}$ improving the bandwidth available in radiant power measurements. These devices are free of the "dead layer" that commonly desensitizes Si photodiodes to ultraviolet radiation, allowing continuous sensitivity from the near infrared into the soft $\mathrm{x}$ rays. The disruption radiometer (DISRAD) diagnostic $^{7}$ for DIII-D, described in this article, employs these devices, allowing measurements on time scales as short as $10^{-5} \mathrm{~s}$. This system has a single line of sight through the plasma, shown in Fig. 1.

A standard diagnostic for radiated power measurements

${ }^{\text {a)} E l e c t r o n i c ~ m a i l: ~ d s g r a y @ u c s d . e d u ~}$ in tokamaks is the foil bolometer. Although the temporal response of the bolometer is limited by heat diffusion to $\sim 10^{-2} \mathrm{~s}$, the bolometer has the advantage of producing a wavelength-independent measure of radiant power. The faster AXUV diode is sensitive across the entire photon energy range from $1.1 \mathrm{eV}$ to about $6 \mathrm{keV}$, but the responsivity varies significantly over this range, as shown in Fig. 2. Ideally, a constant responsivity would be needed to deduce the radiant power (integral over the emission spectrum) from the measured current in the diode. The work described here demonstrates a technique to provide a quantitative measurement of radiated power despite the variation in responsivity in the AXUV diode over the relevant spectral region.

\section{DESIGN}

The responsivity of the AXUV photodiode (amperes of photoconductive current per irradiant watt) is shown in Fig. 2. The flat part of the curve in Fig. 2 above a few hundred $\mathrm{eV}$ photon energy, with responsivity $S=0.275 \mathrm{~A} / \mathrm{W}$, reflects a constant average electron-hole pair creation energy of 3.63 $\mathrm{eV}$ and complete collection of these carriers. The reduced responsivity at lower energies is due to absorption in the passivating layer (typically $6 \mathrm{~nm}$ thick) that covers the diode surface. At low photon energies, the curve is also affected by varying average electron-hole pair creation energy, and at high energies (above a few $\mathrm{keV}$ ) the responsivity falls off as the absorption length begins to surpass the thickness of the epitaxial layer in which the diode is constructed.

In DIII-D plasmas, important emission lines for radiative power loss include Lyman $\alpha$ at $122 \mathrm{~nm}(10.2 \mathrm{eV})$ and the $C_{\text {IV }}$ resonance at $155 \mathrm{~nm}(8.0 \mathrm{eV})$. The responsivity curve 


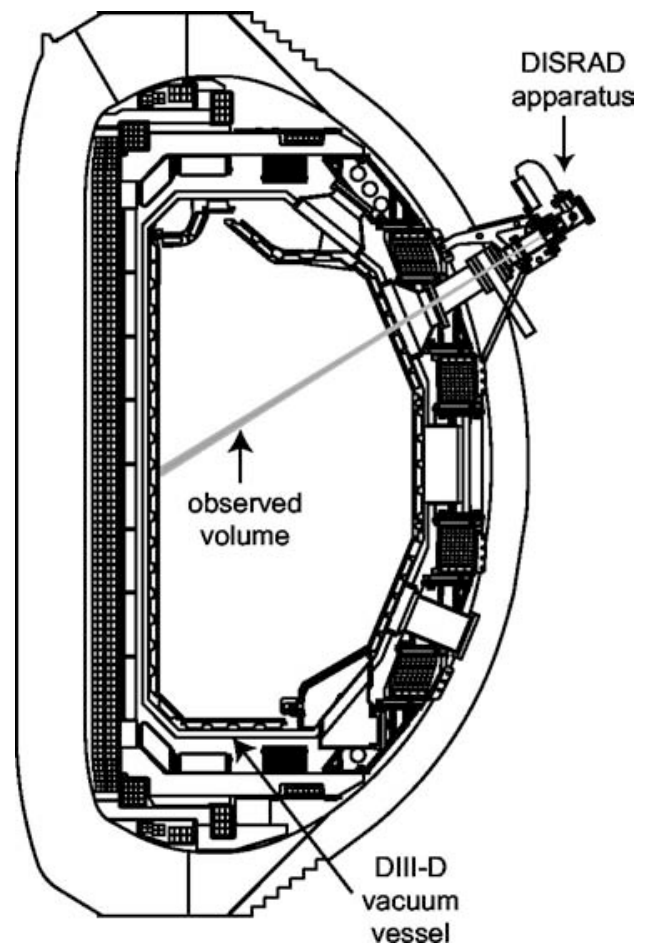

FIG. 1. The DISRAD diagnostic on DIII-D. The three view chords fan out toroidally and have the same path in the poloidal cross section.

varies considerably in this region. The measured diode current can be written as

$$
I=\int S(E) P^{\prime}(E) d E,
$$

where $S(E)$ is the responsivity and $P^{\prime}(E)$ is the spectral power $(\mathrm{W} / \mathrm{eV})$ incident on the detector. The approach taken in this work is to determine averaged responsivity values $S_{\text {eff }}$ for various conditions, by which the current can be expressed as $I=S_{\text {eff }} P$, where the total power $P=\int P^{\prime}(E) d E$ is the actual quantity of interest. This is accomplished by using an AXUV linear array and applying energy selective filtering to different elements. Three array elements view the plasma, essentially in the same spatial region (the views fan out slightly in the toroidal direction). One of the elements has an unfiltered view of the plasma; the other two elements are filtered, as shown in Fig. 3.

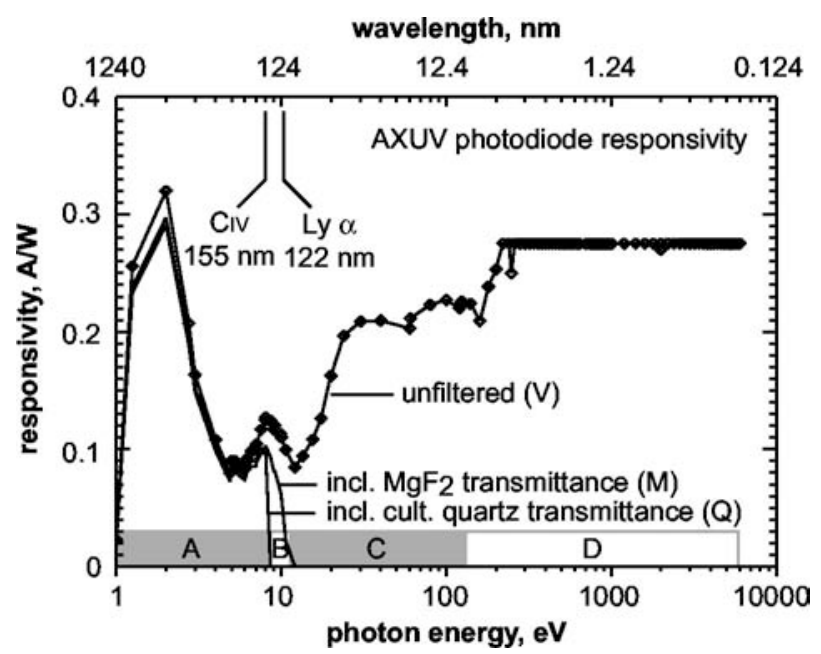

FIG. 2. The responsivity curve of the AXUV photodiode. Curves including the transmittance of the $\mathrm{MgF}_{2}$ and cultured quartz filters are also included. Curves are based on data from manufacturers. The four energy bands referred to in the discussion are indicated.

The filters consist of blank substrates $(2 \mathrm{~mm}$ thick, 12.5 $\mathrm{mm}$ diameter disks) of different materials, each cutting off below a certain wavelength. The filters are from Acton Research Corp. of Acton, MA. Responsivity curves with the filter transmittances folded in are included in Fig. 2. The elements are named "Q," "M," and "V" according to the filter. Element $\mathrm{Q}$ has a cultured quartz filter, which passes at $155 \mathrm{~nm}$ but blocks at $122 \mathrm{~nm}$. Element $\mathrm{M}$ has a $\mathrm{MgF}_{2}$ filter, which passes both $155 \mathrm{~nm}$ and $122 \mathrm{~nm}$ but blocks wavelengths shorter than $\sim 110 \mathrm{~nm}$. Element $\mathrm{V}$ is unfiltered. A blind element is also used to monitor for signal influences unrelated to optical radiation, e.g., hard $\mathrm{x}$ rays or external electronic influences (Fig. 3). Using linear combinations of the currents measured in the three array elements, it is possible to obtain an effective responsivity weighted by the emission spectrum. The additional data and analysis needed to derive this effective responsivity are discussed in Sec. III.

In typical disruption events in DIII-D, DISRAD needs to measure brightnesses up to $\sim 10^{3} \mathrm{~W} \mathrm{~cm}^{-2} \mathrm{sr}^{-1}\left(\sim 10^{5} \mathrm{~J}\right.$ radiated on a $\sim 3 \times 10^{-4}$ s time scale from a $2 \times 10^{7} \mathrm{~cm}^{3}$ volume plasma, viewed along a $10^{2} \mathrm{~cm}$ long chord). The photodiode array views the plasma through a pinhole aperture in a stainless-steel disk of thickness $50 \mu \mathrm{m}$. Pinholes of diam-

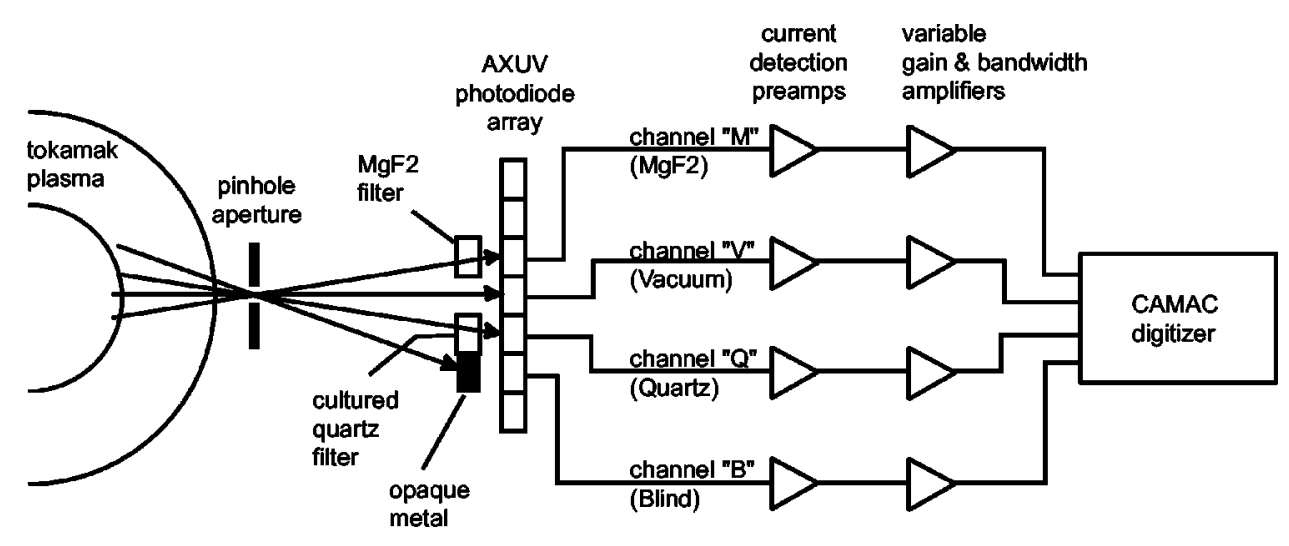

FIG. 3. Overall block schematic diagram of the DISRAD diagnostic. 
eters $100 \mu \mathrm{m}$ and $200 \mu \mathrm{m}$ have been used at various times. Use of a steel foil thinner than $50 \mu \mathrm{m}$ would lead to problems with soft $\mathrm{x}$-ray transmission and possible foil overheating. Because of these same two concerns, a thicker copper foil (125 $\mu \mathrm{m}$ thick, $0.5 \mathrm{~mm}$ hole diameter) is also placed directly in front of the steel foil. The distance from the photodiode array to the pinhole is $50 \mathrm{~mm}$. The use of filters necessitates the introduction of a mask to control the light reaching the photodiode active surface. This mask reduces the exposed area of each element to about one-tenth of its full active area.

Degradation of the responsivity of these photodiodes occurs with cumulative exposure to radiation in the spectral region of strong absorption in the $\mathrm{SiO}_{2}$ passivating layer (9$200 \mathrm{eV}$, roughly). By tailoring the composition of the passivating layer, "AXUV-G" diodes have been developed, which have greatly reduced the degradation rate compared to a plain oxide layer. ${ }^{2}$ An AXUV-G array is used in DISRAD. Still, in measurements from hydrogen plasmas, degradation is a concern because Lyman $\alpha$ is near the most damaging wavelength. It has been demonstrated, however, that the responsivity of the AXUV-G devices can recover fully from degradation if the exposure is turned off for a sufficient length of time. ${ }^{8}$ In the DISRAD diagnostic, any responsivity loss from a DIII-D discharge (estimated to be of order $0.001 \%$ ) is expected to be fully recovered in the time before the next discharge, which is typically $10 \mathrm{~min}$ or more.

Another concern is radiation damage from high-energy neutrons, which is known to occur in photodiodes. Injection of deuterium neutral beams for plasma heating in DIII-D produces $2.5 \mathrm{MeV}$ neutrons, with an average flux of roughly $1 \times 10^{12} \mathrm{~cm}^{-2}$ per operations year in the diagnostic. The principal deleterious effects of neutron exposure on photodiode performance are: (1) reduced responsivity for some photon energy ranges and (2) increased dark current-both related to reduction in the minority carrier lifetime. The rate at which this damage occurs depends on the semiconductor type ( $p$ or $n \mathrm{Si}$ ), resistivity, and construction. ${ }^{9}$ For the devices used here $(25 \mu \mathrm{m}$ thick, $80 \Omega \mathrm{cm}$ epitaxial $p$-Si layer on $p+$ substrate), the damage is not expected to present significant problems at the $1 \times 10^{12} \mathrm{~cm}^{-2}$ neutron fluence level. Therefore, the detector arrays do not need to be replaced more frequently than once per year in this application of the diagnostic.

The currents in the array elements are detected by transimpedance amplifiers (AD745 with $150 \mathrm{k} \Omega \| 10 \mathrm{pF}$ feedback network), with the common anode of the array biased to $-8 \mathrm{~V}$. With bias, the junction capacitance of each $2 \mathrm{~mm}$ $\times 5 \mathrm{~mm}$ element was found to be $\sim 200 \mathrm{pF}$ (this varies from array to array). The cable to the current detection circuit adds another $100 \mathrm{pF}$. The resulting bandwidth is found to be 170 $\mathrm{kHz}$. Variable-gain instrumentation amplifiers following the detection circuits allow measurements in quasisteady (nondisrupting) plasmas, as well as during disruptions, in which the brightness is $\sim 10^{2}$ times higher. The photocurrent detection limit for quasisteady plasmas (noise floor with bandwidth reduced to $30 \mathrm{~Hz}$ ) is $\sim 5 \times 10^{-11} \mathrm{~A}$, while the highest measurable photocurrent during disruptions (instrumentation amplifier input limit) is $3 \times 10^{-6} \mathrm{~A}$. The cable between the diode and the current detection circuit is short $(\sim 0.6 \mathrm{~m})$ to minimize cable capacitance. As a result, the circuit is close to the tokamak magnets and is exposed to large, time-varying magnetic fields. Rejection of pickup from these fields was an important factor in the design of the detection circuits. Avoidance of magnetic induction pickup influences is especially important in measurements from the relatively dim quasisteady plasmas.

Analysis of the DISRAD data is aided by measurements from the "core SPRED" XUV survey spectrometer ${ }^{10}$ in DIII-D. The line of sight of the SPRED diagnostic is in the equatorial plane of the tokamak, passing tangentially through the plasma. Although this view does not coincide with that of DISRAD, both pass through the plasma core and avoid the divertors, so comparison of the two is reasonable. The SPRED has limited time resolution for disruptions; with a minimum integration time of $1 \mathrm{~ms}$, only a few spectra can be acquired in the course of a disruption. Despite these limitations, the spectra provided by the SPRED are important in interpreting the DISRAD measurements. The spectrum allows a weighted average of the responsivity to be calculated over the spectrometer domain (the " $\mathrm{C}$ band" in the following discussion), a region over which the responsivity varies strongly.

\section{ANALYSIS}

In order to interpret the data from our four-channel diode array, we identify four significant photon energy bands: "A" $(1.1$ to $8.3 \mathrm{eV})$, , $\mathrm{B}$ " $(8.3$ to $11 \mathrm{eV})$, , $\mathrm{C}$ " (11 to $130 \mathrm{eV})$, and " $\mathrm{D}$ " $(130 \mathrm{eV}$ to $\sim 6 \mathrm{keV})$. The $\mathrm{A}$ band is seen by all channels $(\mathrm{V}, \mathrm{M}$, and $\mathrm{Q})$, the $\mathrm{B}$ band by channels $\mathrm{V}$ and $\mathrm{M}$ only, and the $\mathrm{C}$ and $\mathrm{D}$ bands are seen only by channel V. The $\mathrm{C}$ band is the domain of the SPRED.

We seek to determine the radiation from each of these four bands independently, using the equations

$$
\left[\begin{array}{c}
I_{\mathrm{Q}} \\
I_{\mathrm{M}} \\
I_{\mathrm{V}}
\end{array}\right]=\left[\begin{array}{cccc}
S_{\mathrm{A}} T_{\mathrm{QA}} & 0 & 0 & 0 \\
S_{\mathrm{A}} T_{\mathrm{MA}} & S_{\mathrm{B}} T_{\mathrm{MB}} & 0 & 0 \\
S_{\mathrm{A}} & S_{\mathrm{B}} & S_{\mathrm{C}} & S_{\mathrm{D}}
\end{array}\right]\left[\begin{array}{c}
P_{\mathrm{A}} \\
P_{\mathrm{B}} \\
P_{\mathrm{C}} \\
P_{\mathrm{D}}
\end{array}\right] .
$$

Here $I_{k}$ is the measured current for channel $k, T_{k x}$ is the transmittance of the channel $k$ filter in band $x, T_{k x} P_{x}$ is the radiant power incident on the diode $\left(P_{x}\right.$ is the unfiltered power), and $S_{x}$ is the AXUV diode responsivity. The A band is assumed to be predominantly $C_{\mathrm{IV}}(8.0 \mathrm{eV})$, so the values $S_{\mathrm{A}}=0.125 \mathrm{~A} / \mathrm{W}, T_{\mathrm{QA}}=0.79$, and $T_{\mathrm{MA}}=0.83$ are used. The B band is assumed to be predominantly Lyman $\alpha(10.2 \mathrm{eV})$, so $S_{\mathrm{B}}=0.11 \mathrm{~A} / \mathrm{W}$ and $T_{\mathrm{MB}}=0.55$ are used. For the $\mathrm{D}$ band, $S_{\mathrm{D}}=0.275 \mathrm{~A} / \mathrm{W}$ is used. For the $\mathrm{C}$ band, $S_{\mathrm{C}}$ is taken as an average over the spectral responsivity curve, using the calibrated SPRED spectrum as a weighting function.

Although there are four photon energy bands of interest, there are only three channels of measured photocurrent. Different assumptions are used to close the system of equations in the two cases of disrupting and quasisteady plasmas.

In disruptions, the system is closed by assuming that $P_{\mathrm{D}}=0$. In the CQ phase, the plasma is too cold to produce radiation in this high-energy band. The assumption is more 


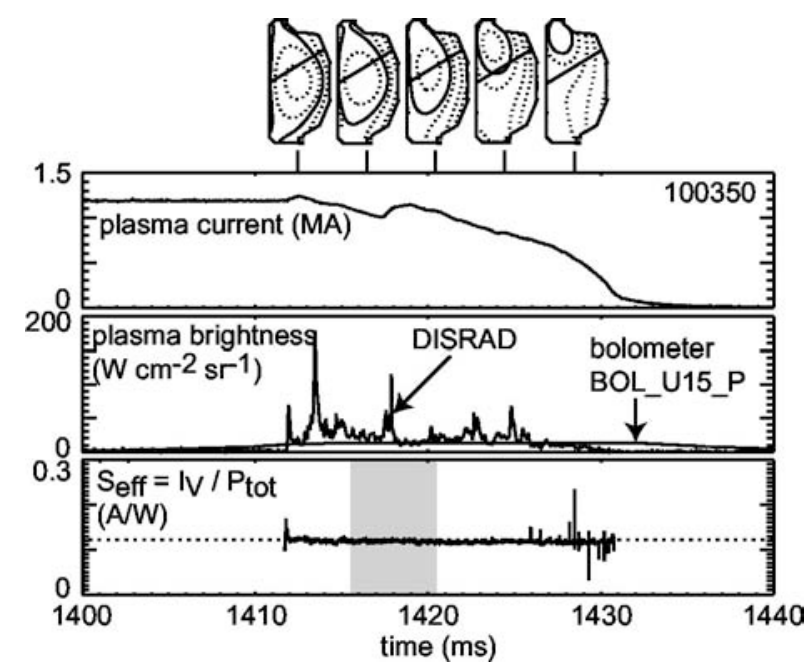

FIG. 4. DISRAD measurements of total brightness $\left(P_{\mathrm{A}}+P_{\mathrm{B}}+P_{\mathrm{C}}\right)$ from a locked-mode disruption in DIII-D. The grayed interval indicates the integration period for the SPRED spectrum shown in Fig. 6. The effective responsivity is found to be $0.12 \mathrm{~A} / \mathrm{W}$ under these conditions. The brightness measured by the equivalent bolometer channel is also shown. Integrating the brightness over the disruption, the DISRAD and bolometer signals give nearly equal emitted energies. The series of flux contour plots was produced using the JFIT code.

questionable in the TQ phase, which is a transition from normal tokamak conditions to the cold CQ conditions.

In quasisteady plasmas, the D-band emission is not negligible. The C-band power can be obtained from the SPRED measurement, using the SPRED absolute brightness calibration. To obtain $P_{\mathrm{C}}$ in DISRAD, the total SPRED brightness ( $\mathrm{W} \mathrm{cm}{ }^{-2} \mathrm{sr}^{-1}$ ) is adjusted to account for the different path length through the plasma. Both viewing chords pass through the plasma core, but since they pass through in different ways, this determination of $P_{\mathrm{C}}$ is approximate. With $P_{\mathrm{A}}$ and $P_{\mathrm{B}}$ taken from $I_{\mathrm{Q}}$ and $I_{\mathrm{M}}$, and with $P_{\mathrm{C}}$ provided by the SPRED, $P_{\mathrm{D}}$ is deduced from $I_{\mathrm{V}}$. The absolute brightness calibration for the SPRED is not needed in the disruption case; in that case, only a relative calibration curve is needed to find the weighted average $S_{\mathrm{C}}$.

In either case, after deducing the radiant powers in the four spectral regions, an effective responsivity can be calculated as $S_{\text {eff }}=I_{\mathrm{V}} /\left(P_{\mathrm{A}}+P_{\mathrm{B}}+P_{\mathrm{C}}+P_{\mathrm{D}}\right)$. As discussed below, we find this figure to be sufficiently reproducible in disruptions that good quantitative results can be obtained from this type of diagnostic without the need for filtering and spectral decomposition, i.e., by simply measuring the unfiltered channel V. In the quasisteady case, $S_{\text {eff }}$ is more variable and the spectral decomposition analysis discussed above is needed to obtain good quantitative results.

\section{RESULTS}

Now, we turn to analysis of data from a DIII-D disruption. The total brightness measured by DISRAD during a locked-mode disruption in DIII-D is shown in Fig. 4. The peak brightness in Fig. 4 corresponds to a photocurrent of about $0.5 \mu \mathrm{A}$ in the diode; this is equivalent to millions of incident photons per microsecond, assuming $\sim 10 \mathrm{eV}$ per

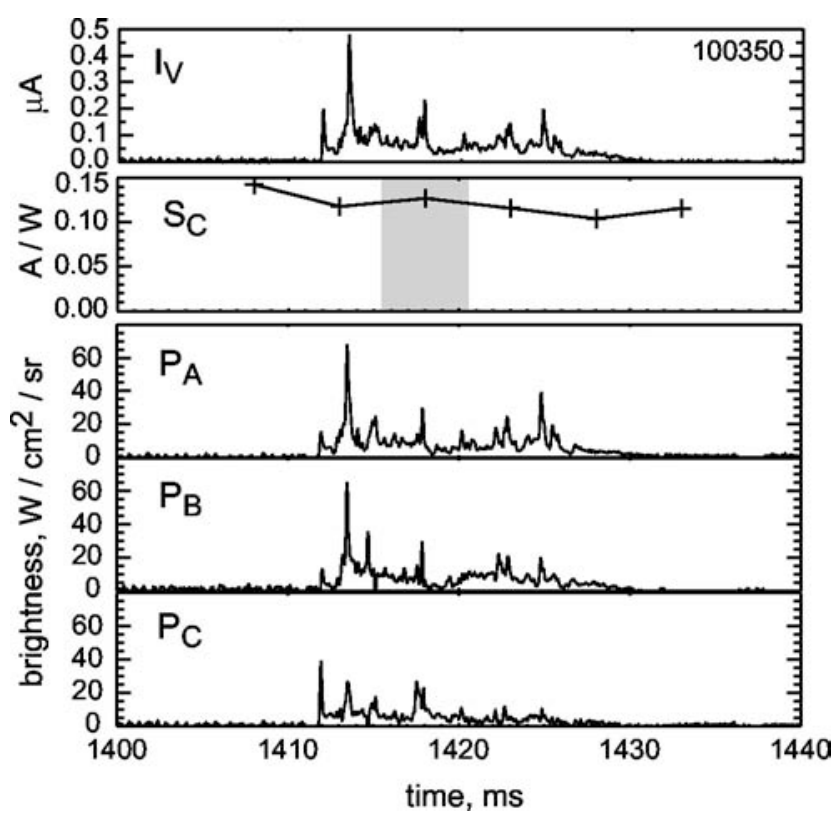

FIG. 5. The A-, B-, and C-band brightness values shown here add up to the total brightness in Fig. 4. The C-band responsivity values are also given, with plot symbols centered in the SPRED integration periods for which the values were calculated. Interpolation of $S_{\mathrm{C}}$ between these points has been used to calculate the $S_{\text {eff }}$ in Fig. 4. The current measured in the unfiltered element is also shown.

photon, so photon statistics are not of serious concern. In Fig. 5, the brightness is displayed separately for the A, B, and $\mathrm{C}$ bands.

The weighted-average responsivity $S_{\text {eff }}$ is found to have a steady value of $0.12 \mathrm{~A} / \mathrm{W}$ during the current quench. This result appears to hold generally for disruptions in DIII-D; while $S_{\text {eff }}$ is not always as constant as in Fig. 4, the $0.12 \mathrm{~A} / \mathrm{W}$ value is valid within $10 \%$. In quasisteady plasmas, $S_{\text {eff }}$ is more variable. The grayed interval in Fig. 4 indicates one of the integrations of the SPRED; in this case, the integration time was set to $5 \mathrm{~ms}$. The averaging of the responsivity in band $\mathrm{C}$ during this integration is illustrated in Fig. 6, which includes the SPRED spectrum from the grayed interval in Fig. 4. At first glance, the weighted average value $S_{\mathrm{C}}$

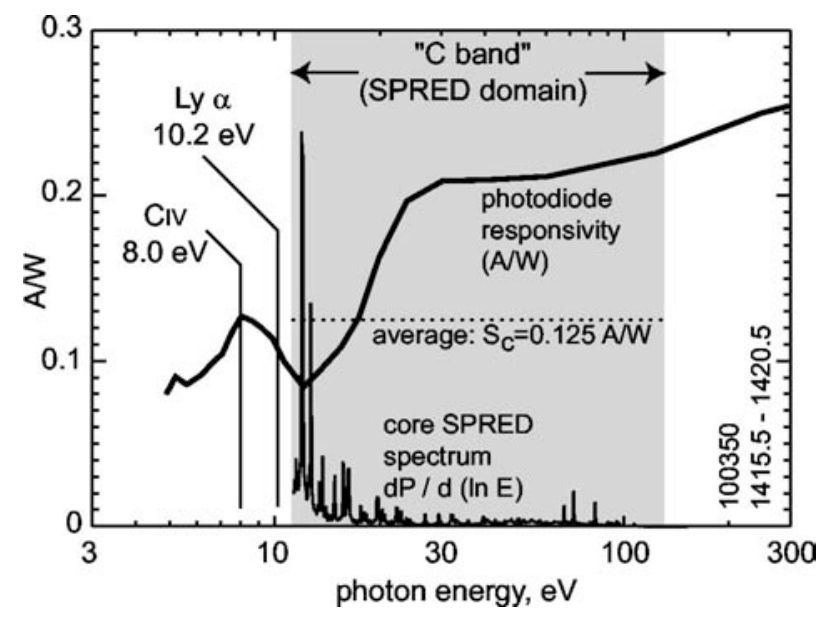

FIG. 6. In band $\mathrm{C}$, the responsivity curve of the AXUV diode is averaged using the SPRED spectrum as a weighting function. The spectrum shown is from the grayed interval in Fig. 4. 


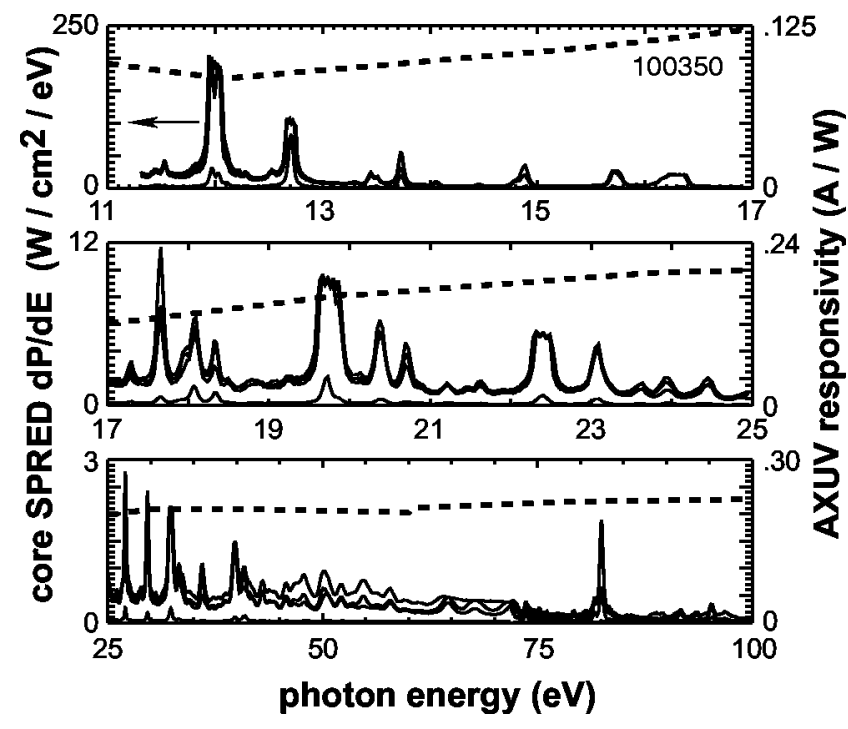

FIG. 7. Four SPRED spectra were acquired in this disruption and are overlaid here with the responsivity curve (broken line). Little difference is observable among the first three spectra, while the fourth is much lower in intensity. The second spectrum corresponds to the grayed interval in Figs. 4 and 5 and appears also in Fig. 6.

$=0.125 \mathrm{~A} / \mathrm{W}$ in Fig. 6 may have the appearance of being too high, as the strongest radiation in band $\mathrm{C}$ is at energies with lower responsivity values. Two factors counterbalance this in the averaging process, however. The first (noting that the responsivity curve $S(E)$ passes through $0.125 \mathrm{~A} / \mathrm{W}$ at about $17 \mathrm{eV}$ photon energy) is that the energy domain in the $\mathrm{C}$ band is much wider above $17 \mathrm{eV}$ than below. The second is that the departure of $S(E)$ above the average is generally larger in the domain above $17 \mathrm{eV}$ than the departure below the average at lower energies.

To illustrate the time evolution of the spectrum in the disruption, four consecutive spectra are shown in Fig. 7. Little difference is found among the first three spectra, while the fourth is less bright as the main plasma has drifted out of the view of the spectrometer. The flux contours in Fig. 4 are produced by the magnetics code JFIT. ${ }^{11}$

A foil bolometer diagnostic is present on DIII-D, ${ }^{12}$ so comparisons with DISRAD are possible. Figure 4 includes the brightness measured in the equivalent bolometer channel, i.e., the channel with a similar $r-\theta$ path as DISRAD, at a different toroidal location. The reason for the appearance of power in this signal before the disruption is that the bolometer signal processing system applies acausal temporal smoothing in the process of deducing power from the rise in foil resistivity. Integrating over the time of the disruption, good agreement is found: DISRAD measures $0.39 \mathrm{~J} \mathrm{~cm}^{-2} \mathrm{sr}^{-1}$, while the bolometer finds $0.41 \mathrm{~J} \mathrm{~cm}^{-2} \mathrm{sr}^{-1}$, thus increasing our confidence in the calibration method for DISRAD.

The brightness measured during a complete quasisteady discharge is shown in Fig. 8. The brightness from the equivalent bolometer channel is shown for comparison. The analysis of the DISRAD data is completely independent of data from the bolometer. The agreement is quite good, although DISRAD tends to read 10\%-20\% lower than the bolometer. In contrast to the disruption case, the effective responsivity

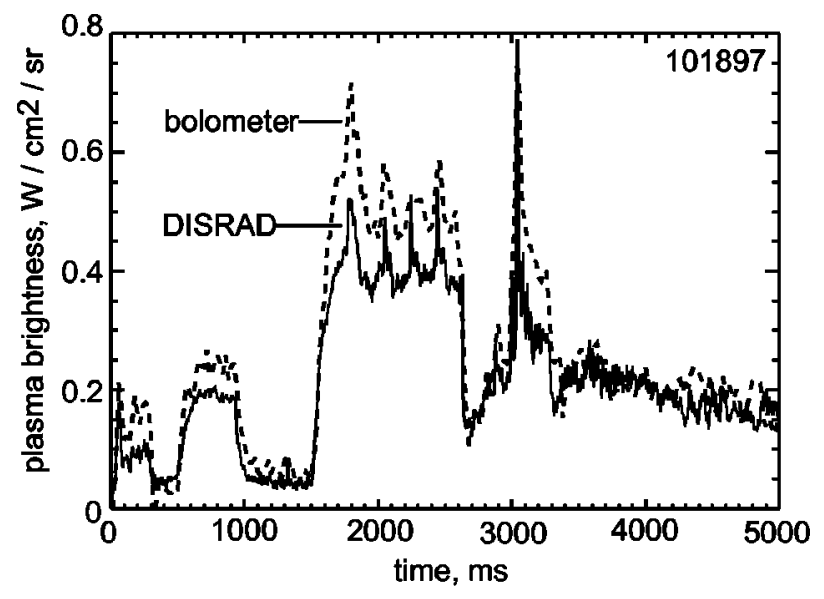

FIG. 8. A comparison of radiated power in a nondisrupting plasma, as determined by DISRAD and the equivalent channel of the bolometer diagnostic. Neutral beam power of $2 \mathrm{MW}$ is applied at $500 \mathrm{~ms}$ and then removed at $900 \mathrm{~ms}$. From $1500 \mathrm{~ms}$ to $5000 \mathrm{~ms}$, about $5 \mathrm{MW}$ of beam power is applied. An H-mode transition occurs at $2600 \mathrm{~ms}$. A pellet is injected at $3000 \mathrm{~ms}$. Agreement is good, although DISRAD tends to read somewhat lower than the bolometer.

deduced from the DISRAD analysis varies considerably in the quasisteady case. Typically, however, the value $S_{\text {eff }}$ $=0.18 \mathrm{~A} / \mathrm{W}$ can be used to obtain a reasonable estimate of the radiated power.

\section{DISCUSSION}

The results shown here demonstrate that the use of the nominal soft $\mathrm{x}$-ray responsivity of $0.275 \mathrm{~A} / \mathrm{W}$ for AXUV photodiodes is inappropriate for measuring total radiated power in plasma devices, such as DIII-D, that have substantial carbon line emissions. In the current quench phase of disruptions in DIII-D, we find the appropriate average to be $S_{\text {eff }}=0.12 \mathrm{~A} / \mathrm{W}$. The strength of these detectors for radiated power measurements in magnetic fusion plasma devices is in response time more than in absolute sensitivity.

Charge exchange $(\mathrm{CX})$ neutral particles may produce significant effects in some AXUV diode applications, depending on the energy of the particles and on whether the geometry allows the particles to reach the detector. In DISRAD, neutrals generally do not play an important role. A neutral that is not reflected at the diode surface or implanted as a neutral may produce signal in the diode, provided that the incident energy is high enough that the ion resulting from the stripping process can penetrate the passivating layer. As with photons, the responsivity to neutrals thus has an energy dependence not exhibited by bolometers. Results from SRIM ${ }^{13,14}$ indicate that the range of deuterium ions in $\mathrm{SiO}_{2}$ exceeds the $6 \mathrm{~nm}$ thickness of the passivating layer for incident energies above $\sim 250 \mathrm{eV}$. At energies above $\sim 2 \mathrm{keV}$, the AXUV responsivity to deuterium ions is comparable to that for photons. ${ }^{15}$

CX neutrals from various sources must be considered separately. CX between thermal edge ions and recycling gas produces neutrals with relatively low energy $\left(\sim T_{i \text {,edge }}\right)$, and hence the diodes have little sensitivity to the associated heat flux. In contrast, bolometers are sensitive to these particles, and the lower reading of DISRAD in Fig. 8 compared to the 
bolometer may be related to this difference between the detectors. In neutral beam heated plasmas, higher-energy neutrals can be produced by $\mathrm{CX}$ between either edge fast ions and recycling gas (energy up to $\sim E_{\text {beam }}$ ), or core ions and beam neutrals $\left(\sim T_{i \text {, core }}\right)$. In DISRAD, fast edge particles do not have a large enough pitch angle to reach the detector (except possibly in sufficiently low-density plasmas). ${ }^{16}$ Core particles may reach the detector if the diagnostic view passes through the neutral beam path and if the CX neutrals are not reionized by the plasma. DISRAD views a neutral beam near the end of its path through the plasma, where generally only a small fraction of the beam neutrals remain.

Used in parallel with standard bolometers, AXUV diodes can provide finer time resolution of power in transients in quasisteady plasmas. In disruptions, accounting for the responsivity is relatively simple, and these devices provide measurements that have heretofore not been available.

\section{ACKNOWLEDGMENTS}

The authors wish to thank R. Korde of International Radiation Detectors (Torrance, CA) for help with many questions about AXUV diode applications, R. T. Snider for guidance in designing the instrument for DIII-D, W. P. West for assistance with SPRED data, A. W. Leonard for help with bolometer data, D. A. Humphreys and P. L. Taylor for JFIT results, and the technical staff at DIII-D for helping to get the diagnostic set up. This work was supported by the
U.S. Department of Energy under Grant No. DE-FG0395ER54294 and Contract No. DE-AC03-99ER54463.

${ }^{1}$ J. A. Wesson, R. D. Gill, M. Hugon, F. C. Schüller, J. A. Snipes, D. J. Ward, D. V. Bartlett, D. J. Campbell, P. A. Duperrex, A. W. Edwards, R. S. Granetz, N. A. O. Gottardi, T. C. Hender, E. Lazzaro, P. J. Lomas, N. Lopes Cardozo, K. F. Mast, M. F. F. Nave, N. A. Salmon, P. Smeulders, P. R. Thomas, B. J. D. Tubbing, M. F. Turner, and A. Weller, Nucl. Fusion 29, 641 (1989).

${ }^{2}$ R. Korde, J. S. Cable, and L. R. Canfield, IEEE Trans. Nucl. Sci. 40, 1655 (1993).

${ }^{3}$ R. J. Maqueda, G. A. Wurden, and E. A. Crawford, Rev. Sci. Instrum. 63, 4717 (1992).

${ }^{4}$ Y. Wen and R. V. Bravenec, Rev. Sci. Instrum. 66, 549 (1995).

${ }^{5}$ R. L. Boivin, J. A. Goetz, E. S. Marmar, J. E. Rice, and J. L. Terry, Rev. Sci. Instrum. 70, 260 (1999).

${ }^{6}$ V. A. Soukhanovskii, D. Stutman, M. Iovea, M. Finkenthal, H. W. Moos, T. Munsat, B. Jones, D. Hoffman, R. Kaita, and R. Majeski, Rev. Sci. Instrum. 72, 737 (2001).

${ }^{7}$ D. S. Gray, S. C. Luckhardt, E. Hollmann, A. G. Kellman, L. Chousal, and G. Gunner, in 27th EPS Conference on Controlled Fusion and Plasma Physics, Budapest, 12-16 June 2000, Europhysics Conference Abstracts 24B, 1681 (2000).

${ }^{8}$ L. R. Canfield, in Vacuum Ultraviolet Spectroscopy II, edited by J. A. R. Samson and D. L. Ederer (Academic, San Diego, 1998), p. 117.

${ }^{9}$ R. Korde, A. Ojha, R. Braasch, and T. C. English, IEEE Trans. Nucl. Sci. 36, 2169 (1989).

${ }^{10}$ R. Wood and S. L. Allen, Rev. Sci. Instrum. 59, 1537 (1988).

${ }^{11}$ D. A. Humphreys and A. G. Kellman, Phys. Plasmas 6, 2742 (1999).

${ }^{12}$ A. W. Leonard, W. H. Meyer, B. Geer, D. M. Behne, and D. N. Hill, Rev. Sci. Instrum. 66, 1201 (1995).

${ }^{13}$ J. F. Ziegler, J. P. Biersack, and U. Littmark, The Stopping and Range of Ions in Solids (Pergamon, New York, 1985).

${ }^{14}$ Code documented at http://www.srim.org

${ }^{15}$ H. O. Funsten, S. M. Ritzau, R. W. Harper, and R. Korde, IEEE Trans. Nucl. Sci. 48, 1785 (2001).

${ }^{16}$ W. W. Heidbrink, T. Beitzel, K. H. Burrell, R. Colchin, C. W. Guldi, and T. Kurki-Suonio, Plasma Phys. Controlled Fusion 43, 373 (2001). 